\title{
Inequalities for the area balance of absolutely continuous functions
}

\author{
Sever S. Dragomir
}

Abstract. We introduce the area balance function associated to a Lebesgue integrable function $f:[a, b] \rightarrow \mathbb{C}$ by

$$
A B_{f}(a, b, \cdot):[a, b] \rightarrow \mathbb{C}, A B_{f}(a, b, x):=\frac{1}{2}\left[\int_{x}^{b} f(t) d t-\int_{a}^{x} f(t) d t\right] .
$$

We show amongst other that, if $f: I \rightarrow \mathbb{C}$ is an absolutely continuous function on the interval $I$ and $[a, b] \subset \stackrel{\circ}{I}$, where $\stackrel{\circ}{I}$ is the interior of $I$ and such that $f^{\prime}$ is of bounded variation on $[a, b]$, then we have the inequality

$$
\begin{gathered}
\left|A B_{f}(a, b, x)-\left(\frac{a+b}{2}-x\right) f(x)-\frac{f^{\prime}(a)+f^{\prime}(b)}{4}\left[\left(x-\frac{a+b}{2}\right)^{2}+\frac{1}{4}(b-a)^{2}\right]\right| \\
\leq \frac{1}{4}\left[\frac{1}{4}(b-a)^{2}+\left(x-\frac{a+b}{2}\right)^{2}\right] \bigvee_{a}^{b}\left(f^{\prime}\right)
\end{gathered}
$$

for any $x \in[a, b]$.

If there exists the real numbers $m, M$ such that

$$
m \leq f^{\prime}(t) \leq M \text { for a.e. } t \in[a, b],
$$

then also

$$
\begin{aligned}
\mid A B_{f}(a, b, x)- & \left(\frac{a+b}{2}-x\right) f(x)-\frac{m+M}{4}\left[\left(x-\frac{a+b}{2}\right)^{2}+\frac{1}{4}(b-a)^{2}\right] \mid \\
& \leq \frac{1}{4}\left[\frac{1}{4}(b-a)^{2}+\left(x-\frac{a+b}{2}\right)^{2}\right](M-m)
\end{aligned}
$$

for any $x \in[a, b]$.

Mathematics Subject Classification (2010): 26D15, 25D10.

Keywords: Functions of bounded variation, Lipschitzian functions, convex functions, integral inequalities. 


\section{Introduction}

For a Lebesgue integrable function $f:[a, b] \rightarrow \mathbb{C}$ and a number $x \in(a, b)$ we can naturally ask how far the integral $\int_{x}^{b} f(t) d t$ is from the integral $\int_{a}^{x} f(t) d t$. If $f$ is nonnegative and continuous on $[a, b]$, then the above question has the geometrical interpretation of comparing the area under the curve generated by $f$ at the right of the point $x$ with the area at the left of $x$. The point $x$ will be called a median point, if

$$
\int_{x}^{b} f(t) d t=\int_{a}^{x} f(t) d t
$$

Due to the above geometrical interpretation, we can introduce the area balance function associated to a Lebesgue integrable function $f:[a, b] \rightarrow \mathbb{C}$ defined as

$$
A B_{f}(a, b, \cdot):[a, b] \rightarrow \mathbb{C}, A B_{f}(a, b, x):=\frac{1}{2}\left[\int_{x}^{b} f(t) d t-\int_{a}^{x} f(t) d t\right] .
$$

Utilising the cumulative function notation $F:[a, b] \rightarrow \mathbb{C}$ given by

$$
F(x):=\int_{a}^{x} f(t) d t
$$

then we observe that

$$
A B_{f}(a, b, x)=\frac{1}{2} F(b)-F(x), x \in[a, b] .
$$

If $f$ is a probability density, i.e. $f$ is nonnegative and $\int_{a}^{b} f(t) d t=1$, then

$$
A B_{f}(a, b, x)=\frac{1}{2}-F(x), x \in[a, b]
$$

In this paper we obtain some inequalities concerning the area balance for absolutely continuous. Applications for differentiable functions whose derivatives are Lipschitzian functions are provided. Bounds involving the Jensen difference

$$
\frac{g(a)+g(b)}{2}-g\left(\frac{a+b}{2}\right)
$$

are also established.

We notice that Jensen difference is closely related to the Hermite-Hadamard type inequalities where various bounds for the quantities

$$
\frac{f(a)+f(b)}{2}-\frac{1}{b-a} \int_{a}^{b} f(t) d t
$$

and

$$
\frac{1}{b-a} \int_{a}^{b} f(t) d t-f\left(\frac{a+b}{2}\right)
$$

are provided, see [1]-[6] and [8]-[18]. 


\section{Preliminary results}

The following representation result holds:

Theorem 2.1. Let $f:[a, b] \rightarrow \mathbb{C}$ be an absolutely continuous function on $[a, b]$. Then we have the representation

$$
\begin{aligned}
A B_{f}(a, b, x) & =\left(\frac{a+b}{2}-x\right) f(x) \\
& +\frac{1}{2}\left[\int_{a}^{x}(t-a) f^{\prime}(t) d t+\int_{x}^{b}(b-t) f^{\prime}(t) d t\right]
\end{aligned}
$$

and

$$
\begin{aligned}
A B_{f}(a, b, x) & =\frac{b f(b)+a f(a)}{2}-\frac{f(b)+f(a)}{2} x \\
& -\frac{1}{2} \int_{a}^{b}|t-x| f^{\prime}(t) d t
\end{aligned}
$$

for any $x \in[a, b]$, where the integrals in the right hand side are taken in the Lebesgue sense.

Proof. Since $f$ is absolutely continuous on $[a, b]$, then $f$ is differentiable almost everywhere (a.e.) on $[a, b]$ and the Lebesgue integrals in the right hand side of the equations (2.1) and (2.2) exist.

Utilising the integration by parts formula for the Lebesgue integral, we have

$$
\begin{aligned}
& \int_{a}^{x}(t-a) f^{\prime}(t) d t+\int_{x}^{b}(b-t) f^{\prime}(t) d t \\
= & \left.(t-a) f(t)\right|_{a} ^{x}-\int_{a}^{x} f(t) d t+\left.(b-t) f(t)\right|_{x} ^{b}+\int_{x}^{b} f(t) d t \\
= & (x-a) f(x)-\int_{a}^{x} f(t) d t-(b-x) f(x)+\int_{x}^{b} f(t) d t \\
= & (2 x-a-b) f(x)+2 A B_{f}(a, b, x)
\end{aligned}
$$

for any $x \in[a, b]$.

Dividing (2.3) by 2 and rearranging the equation, we deduce (2.1).

Integrating by parts, we also have

$$
\begin{aligned}
& \int_{a}^{b}|t-x| f^{\prime}(t) d t \\
= & \int_{a}^{x}(x-t) f^{\prime}(t) d t+\int_{x}^{b}(t-x) f^{\prime}(t) d t \\
= & \left.(x-t) f(t)\right|_{a} ^{x}+\int_{a}^{x} f(t) d t+\left.(t-x) f(t)\right|_{x} ^{b}-\int_{x}^{b} f(t) d t \\
= & -(x-a) f(a)+(b-x) f(b)-2 A B_{f}(a, b, x) \\
= & b f(b)+a f(a)-[f(b)+f(a)] x-2 A B_{f}(a, b, x)
\end{aligned}
$$


for any $x \in[a, b]$.

Dividing (2.4) by 2 and rearranging the equation, we deduce (2.2).

Corollary 2.2. Let $f:[a, b] \rightarrow \mathbb{R}$ be an absolutely continuous function on $[a, b]$. If $f^{\prime}(t) \geq 0$ for a.e. $t \in[a, b]$, then

$$
\begin{aligned}
\frac{b f(b)+a f(a)}{2}-\frac{f(b)+f(a)}{2} x & \geq A B_{f}(a, b, x) \\
& \geq\left(\frac{a+b}{2}-x\right) f(x)
\end{aligned}
$$

for any $x \in[a, b]$.

In particular,

$$
\frac{1}{4}(b-a)[f(b)-f(a)] \geq A B_{f}\left(a, b, \frac{a+b}{2}\right) \geq 0 .
$$

The constant $\frac{1}{4}$ is a best possible constant in the sense that it cannot be replaced by a smaller quantity.

Proof. The inequalities (2.5) follow from the representations (2.1) and (2.2) by taking into account that $f^{\prime}(t) \geq 0$ for a.e. $t \in[a, b]$.

The inequality (2.6) follows by (2.5) for $x=\frac{a+b}{2}$.

Assume that the first inequality in (2.6) holds for a constant $C>0$, i.e.

$$
C(b-a)[f(b)-f(a)] \geq A B_{f}\left(a, b, \frac{a+b}{2}\right)
$$

Consider the function $f_{n}:[-1,1] \rightarrow \mathbb{R}$ given by

$$
f_{n}(t)=\left\{\begin{array}{cc}
0 & \text { if } t \in[-1,0] \\
n t & \text { if } t \in\left(0, \frac{1}{n}\right) \\
1 & \text { if } t \in\left[\frac{1}{n}, 1\right]
\end{array}\right.
$$

where $n \geq 2$, a natural number. This functions is absolutely continuous and $f_{n}^{\prime}(t) \geq 0$ for any $t \in(-1,1)$. We have for $a=-1, b=1$

$$
C(b-a)\left[f_{n}(b)-f_{n}(a)\right]=2 C
$$

and

$$
\begin{aligned}
A B_{f_{n}}\left(a, b, \frac{a+b}{2}\right) & =\frac{1}{2}\left[\int_{0}^{1} f_{n}(t) d t-\int_{-1}^{0} f_{n}(t) d t\right] \\
& =\frac{1}{2}\left(\int_{0}^{\frac{1}{n}} n t d t+\int_{\frac{1}{n}}^{1} 1 d t\right) \\
& =\frac{1}{2}\left(\frac{1}{2 n}+1-\frac{1}{n}\right)=\frac{1}{2}\left(1-\frac{1}{2 n}\right) .
\end{aligned}
$$


Replacing these values in (2.7) we get

$$
2 C \geq \frac{1}{2}\left(1-\frac{1}{2 n}\right)
$$

for any $n \geq 2$.

Taking the limit for $n \rightarrow \infty$ in (2.8) we get $C \geq \frac{1}{4}$, which proves that $\frac{1}{4}$ is best possible in the first inequality in (2.6)

Remark 2.3. Let $f:[a, b] \rightarrow \mathbb{R}$ be an absolutely continuous function on $[a, b]$. If $f^{\prime}(t) \geq 0$ for a.e. $t \in[a, b]$, then $A B_{f}(a, b, x) \geq 0$ for $x \in\left[a, \frac{a+b}{2}\right]\left(\left[\frac{a+b}{2}, b\right]\right)$.

Moreover, if $f(b) \neq-f(a)$ and

$$
\frac{b f(b)+a f(a)}{f(b)+f(a)} \in[a, b]
$$

then

$$
A B_{f}\left(a, b, \frac{b f(b)+a f(a)}{f(b)+f(a)}\right) \leq 0 .
$$

Also, if $f(a), f(b)>0$, then (2.9) holds and the inequality (2.10) is valid.

Corollary 2.4. Let $f:[a, b] \rightarrow \mathbb{C}$ be an absolutely continuous function on $[a, b]$ and $\gamma \in \mathbb{C}$. Then we have the representation

$$
\begin{aligned}
A B_{f}(a, b, x) & =\frac{1}{2} \gamma\left[\left(x-\frac{a+b}{2}\right)^{2}+\frac{1}{4}(b-a)^{2}\right]+\left(\frac{a+b}{2}-x\right) f(x) \\
& +\frac{1}{2}\left[\int_{a}^{x}(t-a)\left(f^{\prime}(t)-\gamma\right) d t+\int_{x}^{b}(b-t)\left(f^{\prime}(t)-\gamma\right) d t\right]
\end{aligned}
$$

and

$$
\begin{aligned}
A B_{f}(a, b, x) & =\frac{b f(b)+a f(a)}{2}-\frac{f(b)+f(a)}{2} x \\
& -\frac{1}{2} \gamma\left[\left(x-\frac{a+b}{2}\right)^{2}+\frac{1}{4}(b-a)^{2}\right] \\
& -\frac{1}{2} \int_{a}^{b}|t-x|\left(f^{\prime}(t)-\gamma\right) d t
\end{aligned}
$$

for any $x \in[a, b]$.

Proof. Let $e(t)=t, t \in[a, b]$. If we write the equality (2.1) for the function $f-\gamma e$ we have

$$
\begin{aligned}
A B_{f-\gamma e}(a, b, x) & =\left(\frac{a+b}{2}-x\right)(f(x)-\gamma x) \\
& +\frac{1}{2}\left[\int_{a}^{x}(t-a)\left(f^{\prime}(t)-\gamma\right) d t+\int_{x}^{b}(b-t)\left(f^{\prime}(t)-\gamma\right) d t\right]
\end{aligned}
$$

for any $x \in[a, b]$. 
Observe that

$$
A B_{f-\gamma e}(a, b, x)=A B_{f}(a, b, x)-\gamma A B_{e}(a, b, x)
$$

and

$$
\begin{aligned}
A B_{e}(a, b, x) & =\frac{1}{2}\left(\int_{x}^{b} t d t-\int_{a}^{x} t d t\right) \\
& =\frac{1}{2}\left(\frac{b^{2}-x^{2}}{2}-\frac{x^{2}-a^{2}}{2}\right)=\frac{1}{2}\left(\frac{a^{2}+b^{2}}{2}-x^{2}\right) .
\end{aligned}
$$

From (2.13) we have

$$
\begin{aligned}
A B_{f}(a, b, x) & =\left(\frac{a+b}{2}-x\right)(f(x)-\gamma x)+\frac{1}{2} \gamma\left(\frac{a^{2}+b^{2}}{2}-x^{2}\right) \\
& +\frac{1}{2}\left[\int_{a}^{x}(t-a)\left(f^{\prime}(t)-\gamma\right) d t+\int_{x}^{b}(b-t)\left(f^{\prime}(t)-\gamma\right) d t\right] \\
& =\left(\frac{a+b}{2}-x\right) f(x)+\frac{1}{2} \gamma\left(\frac{a^{2}+b^{2}}{2}-x^{2}\right)-\gamma\left(\frac{a+b}{2}-x\right) x \\
& +\frac{1}{2}\left[\int_{a}^{x}(t-a)\left(f^{\prime}(t)-\gamma\right) d t+\int_{x}^{b}(b-t)\left(f^{\prime}(t)-\gamma\right) d t\right] \\
& =\frac{1}{2} \gamma\left[x^{2}-(a+b) x+\frac{a^{2}+b^{2}}{2}\right]+\left(\frac{a+b}{2}-x\right) f(x) \\
& +\frac{1}{2}\left[\int_{a}^{x}(t-a)\left(f^{\prime}(t)-\gamma\right) d t+\int_{x}^{b}(b-t)\left(f^{\prime}(t)-\gamma\right) d t\right]
\end{aligned}
$$

for any $x \in[a, b]$.

Since

$$
x^{2}-(a+b) x+\frac{a^{2}+b^{2}}{2}=\left(x-\frac{a+b}{2}\right)^{2}+\frac{1}{4}(b-a)^{2}
$$

then from (2.14) we deduce the desired equality (2.11).

From (2.2) we have

$$
\begin{aligned}
A B_{f-\gamma e}(a, b, x) & =\frac{b f(b)+a f(a)}{2}-\gamma \frac{b^{2}+a^{2}}{2}-\frac{f(b)+f(a)}{2} x+\gamma \frac{a+b}{2} x \\
& -\frac{1}{2} \int_{a}^{b}|t-x|\left(f^{\prime}(t)-\gamma\right) d t
\end{aligned}
$$

and since

$$
A B_{f-\gamma e}(a, b, x)=A B_{f}(a, b, x)-\gamma A B_{e}(a, b, x)
$$


then

$$
\begin{aligned}
A B_{f}(a, b, x) & =\frac{1}{2} \gamma\left(\frac{a^{2}+b^{2}}{2}-x^{2}\right)+\frac{b f(b)+a f(a)}{2} \\
& -\gamma \frac{b^{2}+a^{2}}{2}-\frac{f(b)+f(a)}{2} x+\gamma \frac{a+b}{2} x \\
& -\frac{1}{2} \int_{a}^{b}|t-x|\left(f^{\prime}(t)-\gamma\right) d t \\
& =\frac{b f(b)+a f(a)}{2}-\frac{f(b)+f(a)}{2} x \\
& -\frac{1}{2} \gamma\left[x^{2}-(a+b) x+\frac{a^{2}+b^{2}}{2}\right]-\frac{1}{2} \int_{a}^{b}|t-x|\left(f^{\prime}(t)-\gamma\right) d t
\end{aligned}
$$

which proves the desired equality (2.12).

Remark 2.5. We have the following equalities

$$
\begin{aligned}
A B_{f}\left(a, b, \frac{a+b}{2}\right) & =\frac{1}{8} \gamma(b-a)^{2} \\
& +\frac{1}{2}\left[\int_{a}^{\frac{a+b}{2}}(t-a)\left(f^{\prime}(t)-\gamma\right) d t+\int_{\frac{a+b}{2}}^{b}(b-t)\left(f^{\prime}(t)-\gamma\right) d t\right]
\end{aligned}
$$

and

$$
\begin{aligned}
A B_{f}\left(a, b, \frac{a+b}{2}\right) & =\frac{1}{4}(b-a)[f(b)-f(a)]-\frac{1}{8} \gamma(b-a)^{2} \\
& -\frac{1}{2} \int_{a}^{b}\left|t-\frac{a+b}{2}\right|\left(f^{\prime}(t)-\gamma\right) d t
\end{aligned}
$$

for any $\gamma \in \mathbb{C}$.

\section{Bounds for absolutely continuous functions}

Now, for $\gamma, \Gamma \in \mathbb{C}$ and $[a, b]$ an interval of real numbers, define the sets of complex-valued functions

$$
\bar{U}_{[a, b]}(\gamma, \Gamma):=\{f:[a, b] \rightarrow \mathbb{C} \mid \operatorname{Re}[(\Gamma-f(t))(\overline{f(t)}-\bar{\gamma})] \geq 0 \text { for each } t \in[a, b]\}
$$

and

$$
\bar{\Delta}_{[a, b]}(\gamma, \Gamma):=\left\{f:[a, b] \rightarrow \mathbb{C}|| f(t)-\frac{\gamma+\Gamma}{2}\left|\leq \frac{1}{2}\right| \Gamma-\gamma \mid \text { for each } t \in[a, b]\right\} .
$$

The following representation result may be stated.

Proposition 3.1. For any $\gamma, \Gamma \in \mathbb{C}, \gamma \neq \Gamma$, we have that $\bar{U}_{[a, b]}(\gamma, \Gamma)$ and $\bar{\Delta}_{[a, b]}(\gamma, \Gamma)$ are nonempty, convex and closed sets and

$$
\bar{U}_{[a, b]}(\gamma, \Gamma)=\bar{\Delta}_{[a, b]}(\gamma, \Gamma) .
$$


Proof. We observe that for any $z \in \mathbb{C}$ we have the equivalence

$$
\left|z-\frac{\gamma+\Gamma}{2}\right| \leq \frac{1}{2}|\Gamma-\gamma|
$$

if and only if

$$
\operatorname{Re}[(\Gamma-z)(\bar{z}-\bar{\gamma})] \geq 0
$$

This follows by the equality

$$
\frac{1}{4}|\Gamma-\gamma|^{2}-\left|z-\frac{\gamma+\Gamma}{2}\right|^{2}=\operatorname{Re}[(\Gamma-z)(\bar{z}-\bar{\gamma})]
$$

that holds for any $z \in \mathbb{C}$.

The equality (3.1) is thus a simple consequence of this fact.

On making use of the complex numbers field properties we can also state that:

Corollary 3.2. For any $\gamma, \Gamma \in \mathbb{C}, \gamma \neq \Gamma$, we have that

$$
\begin{aligned}
\bar{U}_{[a, b]}(\gamma, \Gamma)= & \{f:[a, b] \rightarrow \mathbb{C} \mid(\operatorname{Re} \Gamma-\operatorname{Re} f(t))(\operatorname{Re} f(t)-\operatorname{Re} \gamma) \\
& +(\operatorname{Im} \Gamma-\operatorname{Im} f(t))(\operatorname{Im} f(t)-\operatorname{Im} \gamma) \geq 0 \text { for each } t \in[a, b]\} .
\end{aligned}
$$

Now, if we assume that $\operatorname{Re}(\Gamma) \geq \operatorname{Re}(\gamma)$ and $\operatorname{Im}(\Gamma) \geq \operatorname{Im}(\gamma)$, then we can define the following set of functions as well:

$$
\begin{aligned}
& \bar{S}_{[a, b]}(\gamma, \Gamma):=\{f:[a, b] \rightarrow \mathbb{C} \mid \operatorname{Re}(\Gamma) \geq \operatorname{Re} f(t) \geq \operatorname{Re}(\gamma) \\
&\text { and } \operatorname{Im}(\Gamma) \geq \operatorname{Im} f(t) \geq \operatorname{Im}(\gamma) \text { for each } t \in[a, b]\} .
\end{aligned}
$$

One can easily observe that $\bar{S}_{[a, b]}(\gamma, \Gamma)$ is closed, convex and

$$
\emptyset \neq \bar{S}_{[a, b]}(\gamma, \Gamma) \subseteq \bar{U}_{[a, b]}(\gamma, \Gamma) .
$$

Theorem 3.3. Let $f:[a, b] \rightarrow \mathbb{C}$ be an absolutely continuous function on $[a, b]$. If there exists $\gamma, \Gamma \in \mathbb{C}, \gamma \neq \Gamma$ such that $f^{\prime} \in \bar{U}_{[a, b]}(\gamma, \Gamma)$ then

$$
\begin{aligned}
& \mid A B_{f}(a, b, x)-\left(\frac{a+b}{2}-x\right) f(x) \\
& -\frac{\gamma+\Gamma}{4}\left[\left(x-\frac{a+b}{2}\right)^{2}+\frac{1}{4}(b-a)^{2}\right] \mid \\
& \leq \frac{|\Gamma-\gamma|}{4}\left[\frac{1}{4}(b-a)^{2}+\left(x-\frac{a+b}{2}\right)^{2}\right]
\end{aligned}
$$

and

$$
\begin{aligned}
& \mid A B_{f}(a, b, x)-\frac{b f(b)+a f(a)}{2}+\frac{f(b)+f(a)}{2} x \\
& +\frac{\gamma+\Gamma}{4}\left[\left(x-\frac{a+b}{2}\right)^{2}+\frac{1}{4}(b-a)^{2}\right] \mid \\
& \leq \frac{|\Gamma-\gamma|}{4}\left[\frac{1}{4}(b-a)^{2}+\left(x-\frac{a+b}{2}\right)^{2}\right]
\end{aligned}
$$


for any $x \in[a, b]$.

Proof. From the equality (2.11) we have

$$
\begin{aligned}
& A B_{f}(a, b, x) \\
& -\frac{\gamma+\Gamma}{4}\left[\left(x-\frac{a+b}{2}\right)^{2}+\frac{1}{4}(b-a)^{2}\right]-\left(\frac{a+b}{2}-x\right) f(x) \\
& =\frac{1}{2}\left[\int_{a}^{x}(t-a)\left(f^{\prime}(t)-\frac{\gamma+\Gamma}{2}\right) d t+\int_{x}^{b}(b-t)\left(f^{\prime}(t)-\frac{\gamma+\Gamma}{2}\right) d t\right]
\end{aligned}
$$

for any $x \in[a, b]$.

If $f^{\prime} \in \bar{U}_{[a, b]}(\gamma, \Gamma)=\bar{\Delta}_{[a, b]}(\gamma, \Gamma)$, then by taking the modulus in (3.7) we get

$$
\begin{aligned}
& \mid A B_{f}(a, b, x)-\left(\frac{a+b}{2}-x\right) f(x) \\
& -\frac{\gamma+\Gamma}{4}\left[\left(x-\frac{a+b}{2}\right)^{2}+\frac{1}{4}(b-a)^{2}\right] \mid \\
& =\frac{1}{2}\left|\int_{a}^{x}(t-a)\left(f^{\prime}(t)-\frac{\gamma+\Gamma}{2}\right) d t+\int_{x}^{b}(b-t)\left(f^{\prime}(t)-\frac{\gamma+\Gamma}{2}\right) d t\right| \\
& \leq \frac{1}{2}\left[\left|\int_{a}^{x}(t-a)\left(f^{\prime}(t)-\frac{\gamma+\Gamma}{2}\right) d t\right|+\left|\int_{x}^{b}(b-t)\left(f^{\prime}(t)-\frac{\gamma+\Gamma}{2}\right) d t\right|\right] \\
& \leq \frac{1}{2}\left[\int_{a}^{x}(t-a)\left|f^{\prime}(t)-\frac{\gamma+\Gamma}{2}\right| d t+\int_{x}^{b}(b-t)\left|f^{\prime}(t)-\frac{\gamma+\Gamma}{2}\right| d t\right] \\
& \leq \frac{|\Gamma-\gamma|}{4}\left[\int_{a}^{x}(t-a) d t+\int_{x}^{b}(b-t) d t\right] \\
& =\frac{|\Gamma-\gamma|}{4}\left[\frac{(x-a)^{2}+(b-x)^{2}}{2}\right]=\frac{|\Gamma-\gamma|}{4}\left[\frac{1}{4}(b-a)^{2}+\left(x-\frac{a+b}{2}\right)^{2}\right],
\end{aligned}
$$

for any $x \in[a, b]$, which proves the inequality (3.5).

From the equality (2.12) we have

$$
\begin{aligned}
& A B_{f}(a, b, x)-\frac{b f(b)+a f(a)}{2}+\frac{f(b)+f(a)}{2} x \\
& +\frac{\gamma+\Gamma}{4}\left[\left(x-\frac{a+b}{2}\right)^{2}+\frac{1}{4}(b-a)^{2}\right] \\
& =-\frac{1}{2} \int_{a}^{b}|t-x|\left(f^{\prime}(t)-\frac{\gamma+\Gamma}{2}\right) d t
\end{aligned}
$$

for any $x \in[a, b]$.

Taking the modulus in (3.8) and using the fact that

$$
f^{\prime} \in \bar{U}_{[a, b]}(\gamma, \Gamma)=\bar{\Delta}_{[a, b]}(\gamma, \Gamma)
$$


we have

$$
\begin{aligned}
& \mid A B_{f}(a, b, x)-\frac{b f(b)+a f(a)}{2}+\frac{f(b)+f(a)}{2} x \\
& +\frac{\gamma+\Gamma}{4}\left[\left(x-\frac{a+b}{2}\right)^{2}+\frac{1}{4}(b-a)^{2}\right] \mid \\
& \leq \frac{1}{2} \int_{a}^{b}|t-x|\left|f^{\prime}(t)-\frac{\gamma+\Gamma}{2}\right| d t \\
& \leq \frac{|\Gamma-\gamma|}{4} \int_{a}^{b}|t-x| d t=\frac{|\Gamma-\gamma|}{4}\left[\int_{a}^{x}(x-t) d t+\int_{x}^{b}(t-x) d t\right] \\
& =\frac{|\Gamma-\gamma|}{4}\left[\frac{(x-a)^{2}+(b-x)^{2}}{2}\right]=\frac{|\Gamma-\gamma|}{4}\left[\frac{1}{4}(b-a)^{2}+\left(x-\frac{a+b}{2}\right)^{2}\right]
\end{aligned}
$$

for any $x \in[a, b]$, which proves the desired inequality (3.6).

Remark 3.4. Let $f:[a, b] \rightarrow \mathbb{R}$ be an absolutely continuous function on $[a, b]$. If there exists the real numbers $m, M$ such that

$$
m \leq f^{\prime}(t) \leq M \text { for a.e. } t \in[a, b],
$$

then

$$
\begin{aligned}
& \mid A B_{f}(a, b, x)-\left(\frac{a+b}{2}-x\right) f(x) \\
& -\frac{m+M}{4}\left[\left(x-\frac{a+b}{2}\right)^{2}+\frac{1}{4}(b-a)^{2}\right] \mid \\
& \leq \frac{M-m}{4}\left[\frac{1}{4}(b-a)^{2}+\left(x-\frac{a+b}{2}\right)^{2}\right]
\end{aligned}
$$

and

$$
\begin{aligned}
& \mid A B_{f}(a, b, x)-\frac{b f(b)+a f(a)}{2}+\frac{f(b)+f(a)}{2} x \\
& +\frac{m+M}{4}\left[\left(x-\frac{a+b}{2}\right)^{2}+\frac{1}{4}(b-a)^{2}\right] \mid \\
& \leq \frac{M-m}{4}\left[\frac{1}{4}(b-a)^{2}+\left(x-\frac{a+b}{2}\right)^{2}\right]
\end{aligned}
$$

for any $x \in[a, b]$.

Corollary 3.5. With the assumptions of Theorem 3.3 we have

$$
\left|A B_{f}\left(a, b, \frac{a+b}{2}\right)-\frac{\gamma+\Gamma}{16}(b-a)^{2}\right| \leq \frac{|\Gamma-\gamma|}{16}(b-a)^{2}
$$


and

$$
\begin{aligned}
& \left|\frac{1}{4}(b-a)[f(b)-f(a)]-\frac{\gamma+\Gamma}{16}(b-a)^{2}-A B_{f}\left(a, b, \frac{a+b}{2}\right)\right| \\
& \leq \frac{|\Gamma-\gamma|}{16}(b-a)^{2} .
\end{aligned}
$$

Theorem 3.6. Let $f: I \rightarrow \mathbb{R}$ be an absolutely continuous function on the interval $I$ and $[a, b] \subset \stackrel{\circ}{I}$, where $\stackrel{\circ}{I}$ is the interior of $I$ and such that $f^{\prime}$ is of bounded variation on $[a, b]$. Then we have the inequalities

$$
\begin{aligned}
& \mid A B_{f}(a, b, x)-\left(\frac{a+b}{2}-x\right) f(x) \\
& -\frac{f^{\prime}(a)+f^{\prime}(b)}{4}\left[\left(x-\frac{a+b}{2}\right)^{2}+\frac{1}{4}(b-a)^{2}\right] \mid \\
& \leq \frac{1}{4}\left[\frac{1}{4}(b-a)^{2}+\left(x-\frac{a+b}{2}\right)^{2}\right] \bigvee_{a}^{b}\left(f^{\prime}\right)
\end{aligned}
$$

and

$$
\begin{aligned}
& \mid A B_{f}(a, b, x)-\frac{b f(b)+a f(a)}{2}+\frac{f(b)+f(a)}{2} x \\
& +\frac{f^{\prime}(a)+f^{\prime}(b)}{4}\left[\left(x-\frac{a+b}{2}\right)^{2}+\frac{1}{4}(b-a)^{2}\right] \mid \\
& \leq \frac{1}{4}\left[\frac{1}{4}(b-a)^{2}+\left(x-\frac{a+b}{2}\right)^{2}\right] \bigvee_{a}^{b}\left(f^{\prime}\right)
\end{aligned}
$$

for any $x \in[a, b]$.

Proof. From (2.11) for $\gamma=\frac{f^{\prime}(a)+f^{\prime}(b)}{2}$ we have the representation

$$
\begin{aligned}
& A B_{f}(a, b, x) \\
& -\frac{f^{\prime}(a)+f^{\prime}(b)}{4}\left[\left(x-\frac{a+b}{2}\right)^{2}+\frac{1}{4}(b-a)^{2}\right]-\left(\frac{a+b}{2}-x\right) f(x) \\
& =\frac{1}{2}\left[\int_{a}^{x}(t-a)\left(f^{\prime}(t)-\frac{f^{\prime}(a)+f^{\prime}(b)}{2}\right) d t\right. \\
& \left.+\int_{x}^{b}(b-t)\left(f^{\prime}(t)-\frac{f^{\prime}(a)+f^{\prime}(b)}{2}\right) d t\right]
\end{aligned}
$$

for any $x \in[a, b]$. 
Taking the modulus in (3.15) we get

$$
\begin{aligned}
& \mid A B_{f}(a, b, x)-\left(\frac{a+b}{2}-x\right) f(x) \\
& -\frac{f^{\prime}(a)+f^{\prime}(b)}{4}\left[\left(x-\frac{a+b}{2}\right)^{2}+\frac{1}{4}(b-a)^{2}\right] \mid \\
& \leq \frac{1}{2}\left[\int_{a}^{x}(t-a)\left|f^{\prime}(t)-\frac{f^{\prime}(a)+f^{\prime}(b)}{2}\right| d t\right. \\
& \left.+\int_{x}^{b}(b-t)\left|f^{\prime}(t)-\frac{f^{\prime}(a)+f^{\prime}(b)}{2}\right| d t\right]
\end{aligned}
$$

for any $x \in[a, b]$.

For $t \in[a, x]$ we have

$$
\begin{aligned}
\left|f^{\prime}(t)-\frac{f^{\prime}(a)+f^{\prime}(b)}{2}\right| & =\left|\frac{f^{\prime}(t)-f^{\prime}(a)+f^{\prime}(t)-f^{\prime}(b)}{2}\right| \\
& \leq \frac{1}{2}\left[\left|f^{\prime}(t)-f^{\prime}(a)\right|+\left|f^{\prime}(b)-f^{\prime}(t)\right|\right] \\
& \leq \frac{1}{2} \bigvee_{a}^{b}\left(f^{\prime}\right)
\end{aligned}
$$

and similarly, for $t \in[x, b]$ we have

$$
\left|f^{\prime}(t)-\frac{f^{\prime}(a)+f^{\prime}(b)}{2}\right| \leq \frac{1}{2} \bigvee_{a}^{b}\left(f^{\prime}\right)
$$

and then by (3.16) we get

$$
\begin{aligned}
& \mid A B_{f}(a, b, x)-\left(\frac{a+b}{2}-x\right) f(x) \\
& -\frac{f^{\prime}(a)+f^{\prime}(b)}{4}\left[\left(x-\frac{a+b}{2}\right)^{2}+\frac{1}{4}(b-a)^{2}\right] \mid \\
& \leq \frac{1}{4}\left[\int_{a}^{x}(t-a) d t+\int_{x}^{b}(b-t) d t\right] \bigvee_{a}^{b}\left(f^{\prime}\right) \\
& =\frac{1}{4}\left[\frac{1}{4}(b-a)^{2}+\left(x-\frac{a+b}{2}\right)^{2}\right] \bigvee_{a}^{b}\left(f^{\prime}\right)
\end{aligned}
$$

for $t \in[a, b]$, and the inequality (3.13) is proved.

The second inequality goes along a similar way and we omit the details.

Corollary 3.7. With the assumptions of Theorem 3.6 we have

$$
\left|A B_{f}\left(a, b, \frac{a+b}{2}\right)-\frac{f^{\prime}(a)+f^{\prime}(b)}{16}(b-a)^{2}\right| \leq \frac{1}{16}(b-a)^{2} \bigvee_{a}^{b}\left(f^{\prime}\right)
$$


and

$$
\begin{aligned}
& \left|\frac{1}{4}(b-a)[f(b)-f(a)]-\frac{f^{\prime}(a)+f^{\prime}(b)}{16}(b-a)^{2}-A B_{f}\left(a, b, \frac{a+b}{2}\right)\right| \\
& \leq \frac{1}{16}(b-a)^{2} \bigvee_{a}^{b}\left(f^{\prime}\right) .
\end{aligned}
$$

\section{Bounds for Lipschitzian derivatives}

We say that $v$ is Lipschitzian with the constant $L>0$, if

$$
|v(t)-v(s)| \leq L|t-s|
$$

for any $t, s \in[a, b]$.

Theorem 4.1. Let $f: I \rightarrow \mathbb{R}$ be an absolutely continuous function on the interval $I$ and $[a, b] \subset \stackrel{\circ}{I}$, where $\stackrel{\circ}{I}$ is the interior of $I$ and such that $f^{\prime}$ is Lipschitzian with the constant $K>0$ on $[a, b]$. Then we have the inequalities

$$
\begin{aligned}
& \mid A B_{f}(a, b, x)-\left(\frac{a+b}{2}-x\right) f(x) \\
& -\frac{1}{2} f^{\prime}(x)\left[\frac{1}{4}(b-a)^{2}+\left(x-\frac{a+b}{2}\right)^{2}\right] \mid \\
& \leq \frac{1}{12}(b-a) K\left[3\left(x-\frac{a+b}{2}\right)^{2}+\frac{1}{4}(b-a)^{2}\right]
\end{aligned}
$$

for any $x \in[a, b]$.

In particular, we have

$$
\left|A B_{f}\left(a, b, \frac{a+b}{2}\right)-\frac{1}{8} f^{\prime}\left(\frac{a+b}{2}\right)(b-a)^{2}\right| \leq \frac{1}{48} K(b-a)^{3} .
$$

The constant $\frac{1}{48}$ is best possible in (4.2).

Proof. We have from the equality (2.11) that

$$
\begin{aligned}
& A B_{f}(a, b, x) \\
& -\left(\frac{a+b}{2}-x\right) f(x)-\frac{1}{2} f^{\prime}(x)\left[\frac{1}{4}(b-a)^{2}+\left(x-\frac{a+b}{2}\right)^{2}\right] \\
& =\frac{1}{2}\left[\int_{a}^{x}(t-a)\left[f^{\prime}(t)-f^{\prime}(x)\right] d t+\int_{x}^{b}(b-t)\left[f^{\prime}(t)-f^{\prime}(x)\right] d t\right]
\end{aligned}
$$

for any $x \in[a, b]$. 
Taking the modulus on (4.3) we have

$$
\begin{aligned}
& \mid A B_{f}(a, b, x)-\left(\frac{a+b}{2}-x\right) f(x) \\
& -\frac{1}{2} f^{\prime}(x)\left[\frac{1}{4}(b-a)^{2}+\left(x-\frac{a+b}{2}\right)^{2}\right] \mid \\
& \leq \frac{1}{2}\left[\int_{a}^{x}(t-a)\left|f^{\prime}(t)-f^{\prime}(x)\right| d t+\int_{x}^{b}(b-t)\left|f^{\prime}(t)-f^{\prime}(x)\right| d t\right] \\
& \leq \frac{1}{2} K\left[\int_{a}^{x}(t-a)(x-t) d t+\int_{x}^{b}(b-t)(t-x) d t\right]
\end{aligned}
$$

for any $x \in[a, b]$.

Since a simple calculation shows that

$$
\int_{c}^{d}(t-c)(d-t) d t=\frac{1}{6}(d-c)^{3},
$$

then

$$
\begin{aligned}
& \int_{a}^{x}(t-a)(x-t) d t+\int_{x}^{b}(b-t)(t-x) d t \\
& =\frac{1}{6}\left[(x-a)^{3}+(b-x)^{3}\right] \\
& =\frac{1}{6}(b-a)\left[3\left(x-\frac{a+b}{2}\right)^{2}+\frac{1}{4}(b-a)^{2}\right]
\end{aligned}
$$

for any $x \in[a, b]$.

Utilising (4.4) we get the desired inequality (4.1).

Consider the function $f:[a, b] \rightarrow \mathbb{R}$,

Then $f$ is differentiable and

$$
f(t):=\left\{\begin{array}{cc}
-\left(t-\frac{a+b}{2}\right)^{2} & \text { if } t \in\left[a, \frac{a+b}{2}\right) \\
\left(t-\frac{a+b}{2}\right)^{2} & \text { if } t \in\left[\frac{a+b}{2}, b\right] .
\end{array}\right.
$$

$$
\begin{aligned}
f^{\prime}(t) & =\left\{\begin{array}{cl}
-2\left(t-\frac{a+b}{2}\right) & \text { if } t \in\left[a, \frac{a+b}{2}\right) \\
2\left(t-\frac{a+b}{2}\right) & \text { if } t \in\left[\frac{a+b}{2}, b\right] .
\end{array}\right. \\
& =2\left|t-\frac{a+b}{2}\right|
\end{aligned}
$$

for $t \in[a, b]$.

Since

$$
\begin{aligned}
\left|f^{\prime}(t)-f^{\prime}(s)\right| & =2|| t-\frac{a+b}{2}|-| s-\frac{a+b}{2} \| \\
& \leq 2|t-s|
\end{aligned}
$$


for any $t, s \in[a, b]$, we conclude that $f^{\prime}$ is Lipschitzian with the constant $K=2$.

We have

$$
\begin{aligned}
A B_{f}\left(a, b, \frac{a+b}{2}\right) & =\frac{1}{2}\left[\int_{\frac{a+b}{2}}^{b} f(t) d t-\int_{a}^{\frac{a+b}{2}} f(t) d t\right] \\
& =\frac{1}{2}\left[\int_{\frac{a+b}{2}}^{b}\left(t-\frac{a+b}{2}\right)^{2} d t+\int_{a}^{\frac{a+b}{2}}\left(t-\frac{a+b}{2}\right)^{2} d t\right] \\
& =\frac{1}{2} \int_{a}^{b}\left(t-\frac{a+b}{2}\right)^{2} d t=\frac{1}{24}(b-a)^{3} .
\end{aligned}
$$

If we replace these values in (4.2) we get in both sides the same quantity $\frac{1}{24}(b-a)^{3}$.

The following result also holds:

Theorem 4.2. With the assumptions of Theorem 4.1 we have the inequalities

$$
\begin{aligned}
& \mid A B_{f}(a, b, x)-\frac{b f(b)+a f(a)}{2}+\frac{f(b)+f(a)}{2} x \\
& +\frac{1}{2} f^{\prime}(x)\left[\left(x-\frac{a+b}{2}\right)^{2}+\frac{1}{4}(b-a)^{2}\right] \mid \\
& \leq \frac{1}{12}(b-a) K\left[3\left(x-\frac{a+b}{2}\right)^{2}+\frac{1}{4}(b-a)^{2}\right]
\end{aligned}
$$

for any $x \in[a, b]$.

In particular, we have

$$
\begin{aligned}
& \left|\frac{1}{4}(b-a)[f(b)-f(a)]-\frac{1}{8} f^{\prime}\left(\frac{a+b}{2}\right)(b-a)^{2}-A B_{f}\left(a, b, \frac{a+b}{2}\right)\right| \\
& \leq \frac{1}{48} K(b-a)^{3} .
\end{aligned}
$$

The proof is similar to the above Theorem 4.1 and the details are omitted.

\section{Inequalities for $p$-norms}

For a Lebesgue measurable function $f:[c, d] \rightarrow \mathbb{C}$ we introduce the $p$-Lebesgue norms as

$$
\|f\|_{[c, d], p}:=\left(\int_{a}^{b}|f(t)|^{p} d t\right)^{1 / p} \text { if } p \geq 1
$$

and

$$
\|f\|_{[c, d], \infty}:=e s s \sup _{t \in[c, d]}|f(t)|
$$

provided these quantities are finite. We denote $f \in L_{p}[c, d]$ and $f \in L_{\infty}[c, d]$. 
Proposition 5.1. Let $f:[a, b] \rightarrow \mathbb{C}$ be an absolutely continuous function on $[a, b]$. Then we have the inequalities

$$
\begin{aligned}
& \left|A B_{f}(a, b, x)-\left(\frac{a+b}{2}-x\right) f(x)\right| \\
& \leq \frac{1}{2}\left[\int_{a}^{x}(t-a)\left|f^{\prime}(t)\right| d t+\int_{x}^{b}(b-t)\left|f^{\prime}(t)\right| d t\right]:=B_{1}(x)
\end{aligned}
$$

and

$$
\begin{aligned}
& \left|\frac{b f(b)+a f(a)}{2}-\frac{f(b)+f(a)}{2} x-A B_{f}(a, b, x)\right| \\
& \leq \frac{1}{2} \int_{a}^{b}|t-x|\left|f^{\prime}(t)\right| d t:=B_{2}(x)
\end{aligned}
$$

for any $x \in[a, b]$.

Moreover, we have

$$
\begin{aligned}
B_{1}(x) \leq \frac{1}{2} \times \begin{cases}\frac{1}{2}(x-a)^{2}\left\|f^{\prime}\right\|_{[a, x], \infty} & \text { if } f^{\prime} \in L_{\infty}[a, x] \\
\frac{1}{(\alpha+1)^{1 / \alpha}}(x-a)^{1+1 / \alpha}\left\|f^{\prime}\right\|_{[a, x], \beta} & \text { if } f^{\prime} \in L_{\beta}[a, x], \\
\frac{1}{\alpha}+\frac{1}{\beta}=1, \alpha>1 \\
(x-a)\left\|f^{\prime}\right\|_{[a, x], 1}\end{cases} \\
+\frac{1}{2} \times \begin{cases}\frac{1}{2}(b-x)^{2}\left\|f^{\prime}\right\|_{[x, b], \infty} & \text { if } f^{\prime} \in L_{\infty}[x, b] \\
\frac{1}{(\gamma+1)^{1 / \gamma}}(b-x)^{1+1 / \gamma}\left\|f^{\prime}\right\|_{[x, b], \delta} & \text { if } f^{\prime} \in L_{\delta}[x, b] \\
\frac{1}{\gamma}+\frac{1}{\delta}=1, \gamma>1 \\
(b-x)\left\|f^{\prime}\right\|_{[x, b], 1}\end{cases}
\end{aligned}
$$

and

$$
\begin{aligned}
& B_{2}(x) \leq \frac{1}{2} \times \begin{cases}\frac{1}{2}(x-a)^{2}\left\|f^{\prime}\right\|_{[a, x], \infty} & \text { if } f^{\prime} \in L_{\infty}[a, x] \\
\frac{1}{(\alpha+1)^{1 / \alpha}}(x-a)^{1+1 / \alpha}\left\|f^{\prime}\right\|_{[a, x], \beta} & \text { if } f^{\prime} \in L_{\beta}[a, x], \\
(x-a)\left\|f^{\prime}\right\|_{[a, x], 1} & \frac{1}{\alpha}=1, \alpha>1 \\
\left(x-\frac{1}{\beta}\right. & \end{cases} \\
& +\frac{1}{2} \times \begin{cases}\frac{1}{2}(b-x)^{2}\left\|f^{\prime}\right\|_{[x, b], \infty} & \text { if } f^{\prime} \in L_{\infty}[x, b] \\
\frac{1}{(\gamma+1)^{1 / \gamma}}(b-x)^{1+1 / \gamma}\left\|f^{\prime}\right\|_{[x, b], \delta} & \text { if } f^{\prime} \in L_{\delta}[x, b] \\
& \frac{1}{\gamma}+\frac{1}{\delta}=1, \gamma>1 \\
(b-x)\left\|f^{\prime}\right\|_{[x, b], 1} & \end{cases}
\end{aligned}
$$

for any $x \in[a, b]$. 
Proof. From (2.1) and (2.2) we have by taking the modulus

$$
\begin{aligned}
& \left|A B_{f}(a, b, x)-\left(\frac{a+b}{2}-x\right) f(x)\right| \\
& \leq \frac{1}{2}\left[\left|\int_{a}^{x}(t-a) f^{\prime}(t) d t\right|+\left|\int_{x}^{b}(b-t) f^{\prime}(t) d t\right|\right] \\
& \leq \frac{1}{2}\left[\int_{a}^{x}(t-a)\left|f^{\prime}(t)\right| d t+\int_{x}^{b}(b-t)\left|f^{\prime}(t)\right| d t\right]
\end{aligned}
$$

and

$$
\begin{aligned}
& \left|\frac{b f(b)+a f(a)}{2}-\frac{f(b)+f(a)}{2} x-A B_{f}(a, b, x)\right| \\
& \leq \frac{1}{2} \int_{a}^{b}|t-x|\left|f^{\prime}(t)\right| d t \\
& =\frac{1}{2}\left[\int_{a}^{x}(x-t)\left|f^{\prime}(t)\right| d t+\int_{x}^{b}(t-x)\left|f^{\prime}(t)\right| d t\right]
\end{aligned}
$$

for any $x \in[a, b]$.

Using the Hölder inequality we have

$$
\begin{aligned}
& \leq \frac{1}{2} \times \begin{cases}B_{1}(x) & \text { if } f^{\prime} \in L_{\infty}[a, x] \\
\frac{1}{(\alpha+1)^{1 / \alpha}}(x-a)^{1+1 / \alpha}\left\|f^{\prime}\right\|_{[a, x], \beta} & \begin{array}{l}
\text { if } f^{\prime} \in L_{\beta}[a, x], \\
\frac{1}{\alpha}+\frac{1}{\beta}=1, \alpha>1
\end{array} \\
(x-a)\left\|f^{\prime}\right\|_{[a, x], 1} & \text { if } f^{\prime} \in L_{\infty}[x, b]\end{cases} \\
& +\frac{1}{2} \times \begin{cases}\frac{1}{2}(b-x)^{2}\left\|f^{\prime}\right\|_{[x, b], \infty} & \text { if } f^{\prime} \in L_{\delta}[x, b], \\
\frac{1}{(\gamma+1)^{1 / \gamma}}(b-x)^{1+1 / \gamma}\left\|f^{\prime}\right\|_{[x, b], \delta} & \frac{1}{\gamma}+\frac{1}{\delta}=1, \gamma>1 \\
(b-x)\left\|f^{\prime}\right\|_{[x, b], 1}\end{cases}
\end{aligned}
$$

and a similar inequality for $B_{2}$.

Remark 5.2. We observe that

$$
\begin{aligned}
B_{1}(x) & \leq \frac{1}{4}(x-a)^{2}\left\|f^{\prime}\right\|_{[a, x], \infty}+\frac{1}{4}(b-x)^{2}\left\|f^{\prime}\right\|_{[x, b], \infty} \\
& \leq\left[\frac{1}{4}(x-a)^{2}+\frac{1}{4}(b-x)^{2}\right] \max \left\{\left\|f^{\prime}\right\|_{[a, x], \infty},\left\|f^{\prime}\right\|_{[x, b], \infty}\right\} \\
& =\frac{1}{2}\left[\frac{1}{4}(b-a)^{2}+\left(x-\frac{a+b}{2}\right)^{2}\right]\left\|f^{\prime}\right\|_{[a, b], \infty}
\end{aligned}
$$


therefore

$$
\begin{aligned}
& \left|A B_{f}(a, b, x)-\left(\frac{a+b}{2}-x\right) f(x)\right| \\
& \leq \frac{1}{2}\left[\frac{1}{4}(b-a)^{2}+\left(x-\frac{a+b}{2}\right)^{2}\right]\left\|f^{\prime}\right\|_{[a, b], \infty}
\end{aligned}
$$

for any $x \in[a, b]$.

Similarly,

$$
\begin{aligned}
& \left|\frac{b f(b)+a f(a)}{2}-\frac{f(b)+f(a)}{2} x-A B_{f}(a, b, x)\right| \\
& \leq \frac{1}{2}\left[\frac{1}{4}(b-a)^{2}+\left(x-\frac{a+b}{2}\right)^{2}\right]\left\|f^{\prime}\right\|_{[a, b], \infty}
\end{aligned}
$$

for any $x \in[a, b]$.

In particular, we have

$$
\left|A B_{f}\left(a, b, \frac{a+b}{2}\right)\right| \leq \frac{1}{8}(b-a)^{2}\left\|f^{\prime}\right\|_{[a, b], \infty}
$$

and

$$
\left|\frac{1}{4}(b-a)[f(b)-f(a)]-A B_{f}\left(a, b, \frac{a+b}{2}\right)\right| \leq \frac{1}{8}(b-a)^{2}\left\|f^{\prime}\right\|_{[a, b], \infty} .
$$

\section{Applications for twice differentiable functions}

If we write the equalities (2.11) and (2.12) for the function $f=g^{\prime}$, where $g$ : $I \rightarrow \mathbb{R}$ is a differentiable function on the interior of the interval $I$ with the derivative absolutely continuous on $[a, b] \subset \stackrel{\circ}{I}$, then we get

$$
\begin{aligned}
& A B_{g^{\prime}}(a, b, x) \\
& =\frac{1}{2} \gamma\left[\left(x-\frac{a+b}{2}\right)^{2}+\frac{1}{4}(b-a)^{2}\right]+\left(\frac{a+b}{2}-x\right) g^{\prime}(x) \\
& +\frac{1}{2}\left[\int_{a}^{x}(t-a)\left(g^{\prime \prime}(t)-\gamma\right) d t+\int_{x}^{b}(b-t)\left(g^{\prime \prime}(t)-\gamma\right) d t\right]
\end{aligned}
$$

and

$$
\begin{aligned}
A B_{g^{\prime}}(a, b, x) & =\frac{b g^{\prime}(b)+a g^{\prime}(a)}{2}-\frac{g^{\prime}(b)+g^{\prime}(a)}{2} x \\
& -\frac{1}{2} \gamma\left[\left(x-\frac{a+b}{2}\right)^{2}+\frac{1}{4}(b-a)^{2}\right] \\
& -\frac{1}{2} \int_{a}^{b}|t-x|\left(g^{\prime \prime}(t)-\gamma\right) d t
\end{aligned}
$$


and since

$$
A B_{f}(a, b, x)=\frac{1}{2} F(b)-F(x)
$$

where $F(x):=\int_{a}^{x} f(t) d t$, then

$$
\begin{aligned}
A B_{g^{\prime}}(a, b, x) & =\frac{1}{2}[g(b)-g(a)]-g(x)+g(a) \\
& =\frac{g(a)+g(b)}{2}-g(x)
\end{aligned}
$$

and by (6.1) and (6.2) we get the representations

$$
\begin{aligned}
g(x) & =\frac{g(a)+g(b)}{2} \\
& -\frac{1}{2} \gamma\left[\left(x-\frac{a+b}{2}\right)^{2}+\frac{1}{4}(b-a)^{2}\right]-\left(\frac{a+b}{2}-x\right) g^{\prime}(x) \\
& -\frac{1}{2}\left[\int_{a}^{x}(t-a)\left(g^{\prime \prime}(t)-\gamma\right) d t+\int_{x}^{b}(b-t)\left(g^{\prime \prime}(t)-\gamma\right) d t\right]
\end{aligned}
$$

and

$$
\begin{aligned}
g(x) & =\frac{g(a)+g(b)}{2}-\frac{b g^{\prime}(b)+a g^{\prime}(a)}{2}+\frac{g^{\prime}(b)+g^{\prime}(a)}{2} x \\
& +\frac{1}{2} \gamma\left[\left(x-\frac{a+b}{2}\right)^{2}+\frac{1}{4}(b-a)^{2}\right] \\
& +\frac{1}{2} \int_{a}^{b}|t-x|\left(g^{\prime \prime}(t)-\gamma\right) d t
\end{aligned}
$$

for any $x \in[a, b]$.

If we assume that $g^{\prime \prime} \in \bar{U}_{[a, b]}(\psi, \Psi)$ for some $\psi, \Psi \in \mathbb{C}, \psi \neq \Psi$, then, as above, we have the inequalities

$$
\begin{aligned}
& \mid g(x)-\frac{g(a)+g(b)}{2} \\
& +\frac{\psi+\Psi}{4}\left[\left(x-\frac{a+b}{2}\right)^{2}+\frac{1}{4}(b-a)^{2}\right]+\left(\frac{a+b}{2}-x\right) g^{\prime}(x) \mid \\
& \leq \frac{|\Psi-\psi|}{4}\left[\frac{1}{4}(b-a)^{2}+\left(x-\frac{a+b}{2}\right)^{2}\right]
\end{aligned}
$$


and

$$
\begin{aligned}
& \mid g(x)-\frac{g(a)+g(b)}{2}+\frac{b g^{\prime}(b)+a g^{\prime}(a)}{2}-\frac{g^{\prime}(b)+g^{\prime}(a)}{2} x \\
& -\frac{\psi+\Psi}{4}\left[\left(x-\frac{a+b}{2}\right)^{2}+\frac{1}{4}(b-a)^{2}\right] \mid \\
& \leq \frac{|\Psi-\psi|}{4}\left[\frac{1}{4}(b-a)^{2}+\left(x-\frac{a+b}{2}\right)^{2}\right]
\end{aligned}
$$

for any $x \in[a, b]$.

We have the particular inequalities

$$
\begin{aligned}
& \left|g\left(\frac{a+b}{2}\right)-\frac{g(a)+g(b)}{2}+\frac{\psi+\Psi}{16}(b-a)^{2}\right| \\
& \leq \frac{|\Psi-\psi|}{16}(b-a)^{2}
\end{aligned}
$$

and

$$
\begin{aligned}
& \mid g\left(\frac{a+b}{2}\right)-\frac{g(a)+g(b)}{2}+\frac{1}{4}(b-a)\left[g^{\prime}(b)-g^{\prime}(a)\right] \\
& -\frac{\psi+\Psi}{16}(b-a)^{2} \mid \\
& \leq \frac{|\Psi-\psi|}{16}(b-a)^{2}
\end{aligned}
$$

Other similar results may be stated, however we do not present the details here.

Acknowledgement. The author would like to thank the anonymous referee for reading carefully the manuscript and correcting some typos.

\section{References}

[1] Azpeitia, A.G., Convex functions and the Hadamard inequality, Rev. Colombiana Mat., 28(1994), no. 1, 7-12.

[2] Dragomir, S.S., A mapping in connection to Hadamard's inequalities, An. Öster. Akad. Wiss. Math.-Natur., (Wien), 128(1991), 17-20.

[3] Dragomir, S.S., Two mappings in connection to Hadamard's inequalities, J. Math. Anal. Appl., 167(1992), 49-56.

[4] Dragomir, S.S., On Hadamard's inequalities for convex functions, Mat. Balkanica, 6(1992), 215-222.

[5] Dragomir, S.S., An inequality improving the second Hermite-Hadamard inequality for convex functions defined on linear spaces and applications for semi-inner products, J. Inequal. Pure \& Appl. Math., 3(2002), no. 3, Art. 35.

[6] Dragomir, S.S., Bounds for the normalized Jensen functional, Bull. Austral. Math. Soc., 74(3)(2006), 471-476.

[7] Dragomir, S.S., Refinements of the generalised trapezoid and Ostrowski inequalities for functions of bounded variation, Arch. Math. (Basel), 91(2008), no. 5, 450-460. 
[8] Dragomir, S.S., Gomm, I., Bounds for two mappings associated to the HermiteHadamard inequality, Preprint, RGMIA Res. Rep. Coll., 14(2011), to appear.

[9] Dragomir, S.S., Milośević, D.S., Sándor, J., On some refinements of Hadamard's inequalities and applications, Univ. Belgrad, Publ. Elek. Fak. Sci. Math., 4(1993), 21-24.

[10] Dragomir, S.S., Pearce, C.E.M., Selected Topics on Hermite-Hadamard Inequalities and Applications, RGMIA Monographs, 2000.

[11] Guessab, A., Schmeisser, G., Sharp integral inequalities of the Hermite-Hadamard type, J. Approx. Theory, 115(2002), no. 2, 260-288.

[12] Kilianty, E., Dragomir, S.S., Hermite-Hadamard's inequality and the $p$-HH-norm on the Cartesian product of two copies of a normed space, Math. Inequal. Appl., 13(2010), no. $1,1-32$.

[13] Merkle, M., Remarks on Ostrowski's and Hadamard's inequality, Univ. Beograd. Publ. Elektrotehn. Fak. Ser. Mat., 10(1999), 113-117.

[14] Pearce, C.E.M., Rubinov, A.M., P-functions, quasi-convex functions, and Hadamard type inequalities, J. Math. Anal. Appl., 240 (1999), no. 1, 92-104.

[15] Pečarić, J., Vukelić, A., Hadamard and Dragomir-Agarwal inequalities, the Euler formulae and convex functions, Functional Equations, Inequalities and Applications, 105-137, Kluwer Acad. Publ., Dordrecht, 2003.

[16] Toader, G., Superadditivity and Hermite-Hadamard's inequalities, Studia Univ. BabesBolyai Math., 39(1994), no. 2, 27-32.

[17] Yang, G.-S., Hong, M.-C., A note on Hadamard's inequality, Tamkang J. Math., 28(1997), no. 1, 33-37.

[18] Yang, G.-S., Tseng, K.-L., On certain integral inequalities related to Hermite-Hadamard inequalities, J. Math. Anal. Appl., 239(1999), no. 1, 180-187.

Sever S. Dragomir

Mathematics, College of Engineering \& Science

Victoria University, PO Box 14428

Melbourne City, MC 8001, Australia

e-mail: sever.dragomir@vu.edu.au

DST-NRF Centre of Excellence in the Mathematical and Statistical Sciences

School of Computer Science and Applied Mathematics

University of the Witwatersrand

Private Bag 3, Johannesburg 2050, South Africa 